\title{
Wireless and Battery-less Sensor Using RF Energy Harvesting
}

\author{
Christian Merz ${ }^{1}$, Gerald Kupris $^{2}$, Maximilian Niedernhuber ${ }^{3}$ \\ Deggendorf Institute of Technology, Edlmairstr. 6 + 8, 94469 Deggendorf, Germany \\ ${ }^{1}$ christian.merz@th-deg.de \\ 2 gerald.kupris@th-deg.de \\ ${ }^{3}$ maximilian.niedernhuber@th-deg.de
}

\begin{abstract}
The contribution introduces a RF energy harvesting circuit which can be used to power a wireless and battery-less sensor system. The sensor can be powered wirelessly over a distance of about two meters. The basic principles of RF energy harvesting are explained, it is shown how the system can be designed and special considerations are discussed. The proposed RF energy harvesting system operates at a frequency of $866.6 \mathrm{MHz}$, so that far field propagation is present. The system consists of an antenna, a matching circuit, a RF-to-DC conversion circuit and a power management module, including a storage capacitor, a comparator and a DC-to-DC converter. The harvester produces a pulsed output voltage of $1.8 \mathrm{~V}$ at an input power of at least $-6 \mathrm{dBm}$.
\end{abstract}

Key words: RF Energy Harvesting, Wireless Power Transfer, Battery-less Sensor, RF-to-DC Conversion, Electromagnetic Far Field Propagation

\section{Introduction}

Energy harvesting is the process of capturing, conversion and storing of energy from the environment to supply low power devices or saving the energy for later use. There are many different types of energy harvesting sources, e.g. solar, vibration, temperature or electromagnetic waves. Of these sources, electromagnetic waves provide by far the least power density, which is indicated by table 1. [1]

Tab. 1: Different energy harvesting sources.

\begin{tabular}{|c|c|c|}
\hline Source & Technology & $\begin{array}{c}\text { Power } \\
\text { Density }\end{array}$ \\
\hline Solar & $\begin{array}{c}\text { Photovoltaic } \\
\text { Cell }\end{array}$ & $\begin{array}{c}100 \\
\mathrm{~mW} / \mathrm{cm}^{2}\end{array}$ \\
\hline Vibration & $\begin{array}{c}\text { Piezoelectric } \\
\text { Element }\end{array}$ & $\begin{array}{c}800 \\
\mu \mathrm{W} / \mathrm{cm}^{2}\end{array}$ \\
\hline Temperature & $\begin{array}{c}\text { Thermal } \\
\text { Generator }\end{array}$ & $\begin{array}{c}60 \\
\mu \mathrm{W} / \mathrm{cm}^{2}\end{array}$ \\
\hline $\begin{array}{c}\text { Electromagnetic } \\
\text { Waves }\end{array}$ & Antenna & $\begin{array}{c}< \\
1 \mu \mathrm{W} / \mathrm{cm}^{2}\end{array}$ \\
\hline
\end{tabular}

Because of the fact that the power density of electromagnetic waves is very low, the energy feed-in and conversion has to be done very efficiently. The feed-in is performed by an antenna, which captures the incident electromagnetic waves and transfers the resulting AC voltage to the harvester circuit. Between the antenna and the energy conversion circuit there is the matching network. On the one hand, it has the task to ensure the maximum power delivery from the antenna to the remaining circuit and on the other hand it minimizes the signal reflections at the interface of the antenna and the matching circuit. The energy conversion circuit converts the RF power, which is delivered by the antenna, into DC voltage by using a cascaded Greinacher circuit. After the rectification, the resulting DC voltage is stored at a capacitor. The energy at this storage capacitor gets accumulated until an upper threshold voltage is reached. After exceeding this voltage, the storage capacitor discharges until a lower threshold voltage is reached. This charging and discharging of the capacitor is triggered by a comparator, which compares the voltage at the storage capacitor with an internal reference voltage and outputs a digital voltage indicating which one of the compared voltages is higher. The output of the comparator switches a DC-toDC converter on and off, which increases the DC voltage level at the storage capacitor to another adjustable level, e.g. $1.8 \mathrm{~V}$. Fig. 1 shows the components of the proposed RF energy harvesting system. 


\section{Comparator}

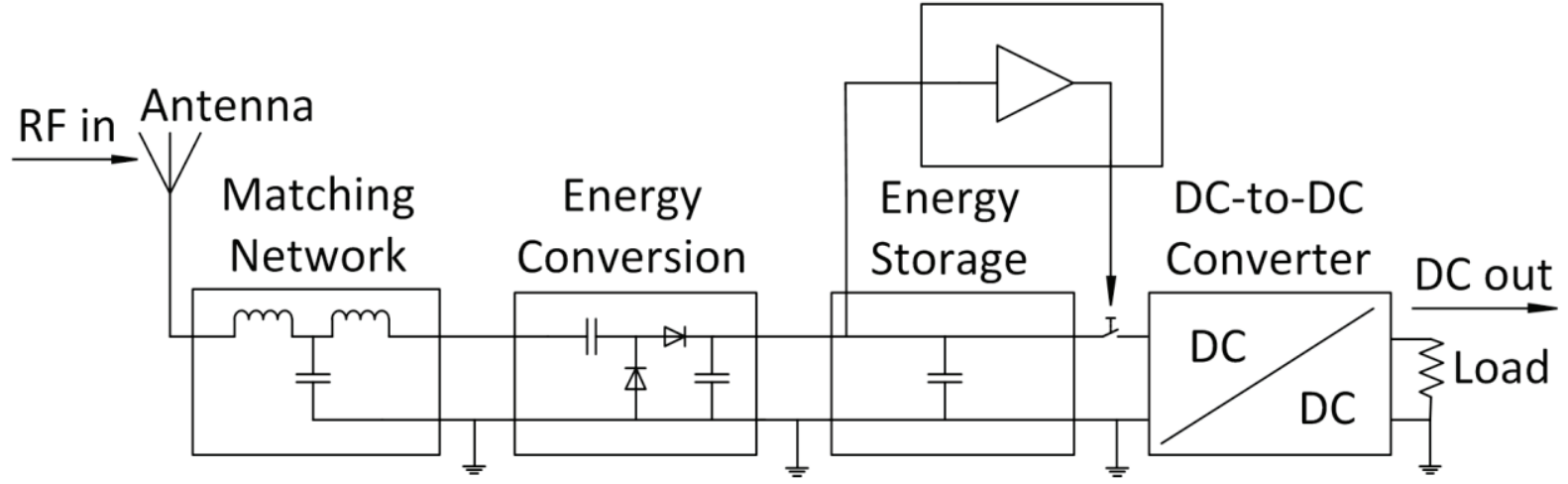

Fig. 1. Diagram of the proposed RF energy harvesting system.

\section{Preconsiderations}

The selection of the frequency band and the determination of the expected input power are very important aspects that must be dealt with before starting the design of the RF energy harvesting system. For energy harvesting purposes, four frequencies are mainly used in today's industry for different applications. The frequencies have a major influence on the design and on the transmission behavior of the harvesting system. The frequencies are $125 \mathrm{kHz}, 13.56 \mathrm{MHz}, 868 \mathrm{MHz}$ and $2.4 \mathrm{GHz}$. These frequencies are allowed by the ETSI to be used without permission. The different frequencies lead to various coupling mechanisms at distances of several meters. At the frequencies of $125 \mathrm{kHz}$ and $13.56 \mathrm{MHz}$, mostly inductive coupling is used for power transmission. The frequencies of $868 \mathrm{MHz}$ and $2.4 \mathrm{GHz}$ typically are coupled electromagnetically. These coupling mechanisms depend on the field region at which the power transfer occurs. The two field regions are called near-field and far-field. The inductive coupling only occurs at the near-field region and the electromagnetic coupling only takes place at the far-field region. If the distance $d$ of the sender and the receiver of the transmission is below $N /(2 \pi)$, the near-field is present. Above this distance, the region is considered as the far-field. Within the near-field region, the magnetic field strength is dominant and decreases according to $1 / \mathrm{d}^{3}$. At the farfield, the magnetic and electric field are in phase and create an electromagnetic wave. The field strengths are decreasing within this region according to $1 / \mathrm{d}$. Because of this, the use of far-field propagation is preferred to nearfield propagation for long range applications. The frequency bands are divided into subbands, where different maximal field strengths or transmission powers are allowed. The $868 \mathrm{MHz}$ band has for example the subband of $865.6 \mathrm{MHz}-867.6 \mathrm{MHz}$, where a transmission power of 2 Watt (ERP) is allowed without restrictions. Because of this, we use the frequency of $866.6 \mathrm{MHz}$ for the power transfer, which is the middle of the subband. At the different frequencies, different antenna types are typically used to capture the energy. At $125 \mathrm{kHz}$ and $13.56 \mathrm{MHz}$ mostly antenna coils are used. At $868 \mathrm{MHz}$ and $2.4 \mathrm{GHz}$ mainly PCB-, chip-, patch- or monopole antennas are utilized depending on the application.

To calculate the incident power at the antenna at a certain distance $\mathrm{R}$ to the sender, the Friis transmission equation has to be used. It relates the received signal power $\left(P_{r}\right)$ and the transmitted signal power $\left(P_{t}\right)$ as

$$
P_{r}=P_{t} G_{t} G_{r}\left(\frac{\lambda}{4 \pi R}\right)^{2}
$$

$G_{t}$ and $G_{r}$ are the antenna gains of the transmitting and the receiving antenna and $\lambda$ is the wavelength of the transmitted signal. The Friis formula is only valid for the following four conditions.

First, the transmission has to take place in the far-field region at free space conditions. Second, the transmitting and receiving antenna must be correctly aligned and polarized. Third, the bandwidth of the transmission has to be so narrow that one value for the wavelength can be assumed. Four, the two antennas and their transmission lines are conjugate matched, so that no losses occur due to mismatching.

\section{Antenna}

The antenna has the task to harvest the incident electromagnetic waves that are propagated by a RF transmitter. On the one hand, the gain of the antenna should be as high as possible to increase the received power. 
On the other hand, the antenna should be as isotropical as possible, so that the direction at which the electromagnetic waves are captured does not influence the harvesting very much. Because the two features cannot be achieved at the same time, a compromise has to be found. A quarter-wave monopole antenna is the best compromise, because it has a high gain of $2.15 \mathrm{dBi}$ and an approximately isotropic antenna diagram, except at the antenna axis. The length of the quarter wave monopole at $866.6 \mathrm{MHz}$ is $8.655 \mathrm{~cm}$. The impedance of the antenna is $50 \Omega$, which is the same value typical RF measurement devices have.

\section{Matching Circuit}

The matching circuit is needed to ensure the maximum power delivery from the antenna to the remaining circuit and to minimize signal reflections. A T-match circuit is used to accomplish this task. It consists of two series inductors and one parallel variable capacitor.

The first step of the matching procedure is to determine the impedance of the harvesting circuit without the antenna and matching circuit. This can be performed by simulation or experimentally by using a network analyzer. In this work, the impedance has been determined experimentally. The impedance of the circuit does not only depend on the frequency, but also on the input power because of the diodes, which are nonlinear devices. The matching can be performed at one particular frequency and input power. The impedance of the circuit has been measured at a frequency at $866.6 \mathrm{MHz}$ and an input power of $-6 \mathrm{dBm}$, which is the lowest power at which the harvester begins to work. The measured impedance has the value of $97 \Omega+\mathrm{j} 96 \Omega$. This impedance has to be matched to the $50 \Omega$ of the antenna.

The second step of the matching procedure is the calculation of the reactive elements of the T-match circuit. For this calculation, the quality factor $Q$ is a very important parameter. He describes the broadband behavior of the matching and is defined as the relation of the center frequency $f_{0}$ (in our case: 866.6 MHz) and the frequency bandwidth $\Delta f=f_{2}-f_{1}$, where $f_{2}$ and $f_{1}$ are the frequencies where the spectrum of the circuit is reduced to $3 \mathrm{~dB}$ compared to $f_{0}$. For our calculations, we use a $Q$ factor of 3. A T-match network can be interpreted as two back-to-back L-type networks with a common virtual resistance $\mathrm{R}$, which is shown in fig. 2 .

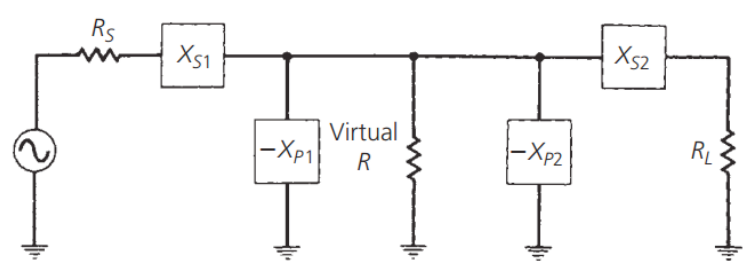

Fig. 2. T-match circuit shown as two back-to-back Lnetworks with common virtual resistor $R$. [2]

The virtual resistance $R$ can be calculated using eq. (2), where $R_{s}=50 \Omega$ is the impedance of the antenna.

$$
R=R_{s}\left(Q^{2}+1\right)=50 \Omega\left(3^{2}+1\right)=500 \Omega
$$

The first serial reactance $X_{s 1}$ can be computed with the following formula.

$X_{s 1}=Q R_{S}=3 \cdot 50 \Omega=150 \Omega$

The first parallel reactance $X_{p 1}$ can be calculated as follows.

$$
X_{p 1}=\frac{R}{Q}=\frac{500 \Omega}{3}=166.7 \Omega
$$

For the L-network at the load end, the quality factor $Q_{2}$ is defined by the virtual resistor $\mathrm{R}$ and the load resistor $R_{L}$ as:

$$
Q_{2}=\sqrt{\frac{R}{R_{L}}-1}=\sqrt{\frac{500 \Omega}{97 \Omega}-1}=2.038
$$

With this value, the second parallel reactance $X_{p 2}$ can be determined as follows.

$$
X_{p 2}=\frac{R}{Q_{2}}=\frac{500 \Omega}{2.038}=245.3 \Omega
$$

The combined equivalent parallel reactance $X_{p}$ can be calculated with eq. (7).

$$
X_{p}=\frac{1}{\frac{1}{X_{p 1}}+\frac{1}{X_{p 2}}}=\frac{1}{\frac{1}{166.7 \Omega}+\frac{1}{245.3 \Omega}}
$$

$=99.23 \Omega$ 
The second serial reactance $X_{s 2}$ can be calculated using the following equation.

$X_{s 2}=Q_{2} R_{L}-X_{L}=2.038 .97 \Omega-96 \Omega$

$=101.69 \Omega$

Finally, the values of the reactive elements $L_{1}$, $L_{2}$ and $C_{\text {var }}$ can be calculated with the following three formulas.

$$
\begin{aligned}
& L_{1}=\frac{X_{s 1}}{2 \pi \cdot f_{0}}=\frac{150 \Omega}{2 \pi \cdot 866.6 \cdot 10^{6} \frac{1}{s}} \\
& =27.5 n H \\
& L_{2}=\frac{X_{s 2}}{2 \pi \cdot f_{0}}=\frac{101.69 \Omega}{2 \pi \cdot 866.6 \cdot 10^{6} \frac{1}{s}} \\
& =18.7 n H \\
& C_{\mathrm{var}}=\frac{1}{2 \pi \cdot f_{0} \cdot X_{p}} \\
& =\frac{1}{2 \pi \cdot 866.6 \cdot 10^{6} \frac{1}{s} \cdot 99.23 \Omega}=1.85 \mathrm{pF}
\end{aligned}
$$

For $L_{1}$ and $L_{2}$ the commercial available values $27 \mathrm{nH}$ and $18 \mathrm{nH}$ have been used.
$C_{\text {var }}$ is a variable capacitor with the range of $1.4 \mathrm{pF}$ to $3 \mathrm{pF}$ so that device tolerances, measurement uncertainties and inductive and capacitive influences caused by the microstrip lines can be compensated. The matching with these components lead to a good match at $-6 \mathrm{dBm}$. Fig. 3 depicts a smith chart that shows the measured impedance of the matched harvester circuit at the antenna input port from $-8 \mathrm{dBm}$ to $8 \mathrm{dBm}$.

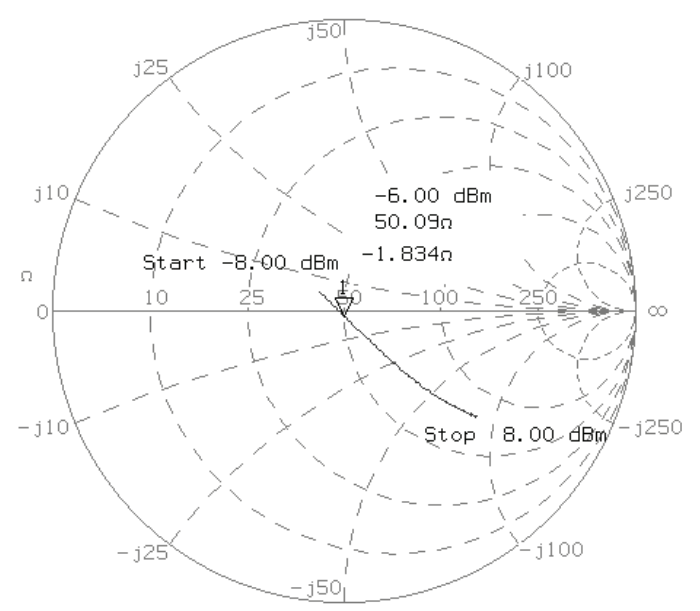

CW $866.600 \mathrm{MHz}$

Fig. 3. Impedance of the matched harvester in dependence on the input power.

Due to the matching, the voltage reflection coefficient decreases to approximately $2 \%$ at an input power of $-6 \mathrm{dBm}$. This means, that $98 \%$ of the power which is captured by the antenna gets delivered to the rest of the harvester circuit. Fig. 4 shows the voltage coefficient in dependence to the input power from $-8 \mathrm{dBm}$ to $8 \mathrm{dBm}$.

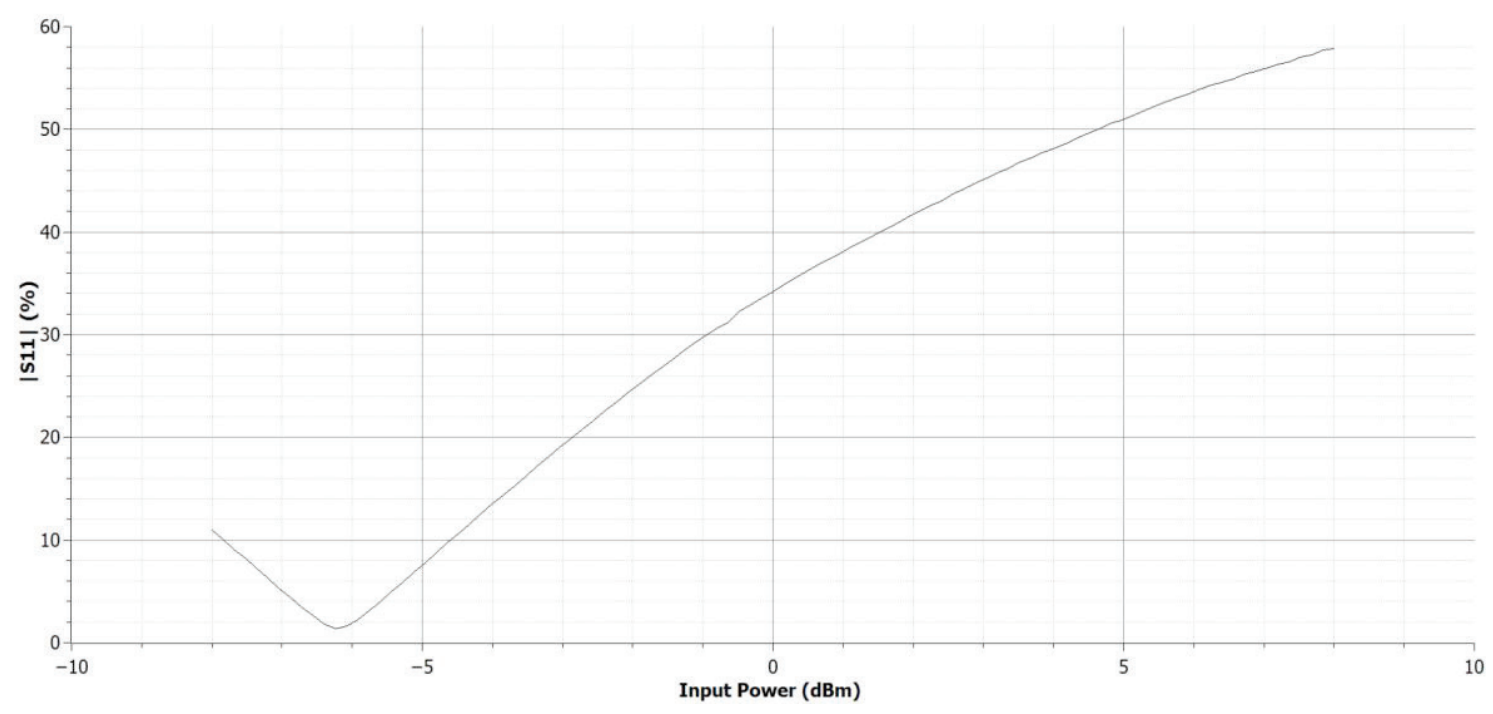

Fig. 4. Voltage reflection coefficient over input power. 


\section{Rectifier Circuit}

To convert the electromagnetic waves into DC voltage, a rectifier circuit is needed. Since the level of the collected power is very low, the rectifier circuit is based on a cascaded voltage multiplier circuit. To accomplish an effective rectification with an acceptable output DC voltage, a 7-stage cascaded Greinacher circuit is used. The circuit not only rectifies the incoming signal but also multiplies the peak amplitude. With increasing stages, the output DC voltage gets higher, but the losses also increase with each stage. Fig. 5 shows a single stage Greinacher circuit.

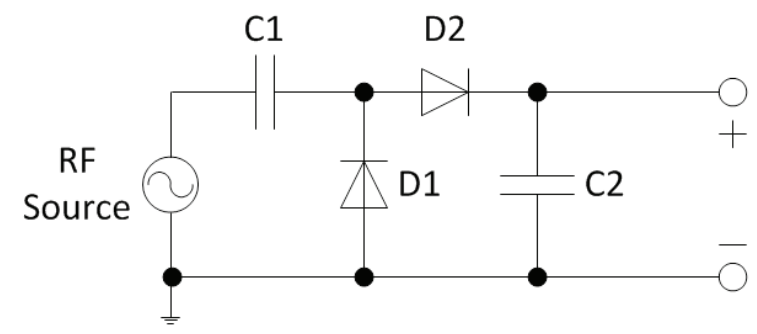

Fig. 5. Single stage Greinacher circuit.

Fig. 5 composes the elementary stage of the rectifier circuit where $C_{1}(2.2 \mathrm{nF})$ and $D_{1}$ form a negative clamp and $C_{2}(2.2 \mathrm{nF})$ and $D_{2}$ achieve peak rectification. $C_{2}$ smoothes the output voltage and acts as a stage storage capacitor. The choice of the diodes is a very important aspect of the rectifier design and is critical for the overall performance of the harvester circuit. Because the harvester circuit operates at a frequency of $866.6 \mathrm{MHz}$ and an input power of $-6 \mathrm{dBm}$, the diodes should have a very fast switching time and a very low turn on voltage. These requirements can be achieved by using Schottky diodes, which use a metalsemiconductor junction instead of a semiconductor-semiconductor junction. This allows the junction to operate much faster and performs a very low forward voltage drop. The Schottky diodes HSMS-285P from Avago Technologies have been selected. They have a maximum forward voltage of $150 \mathrm{mV}$.

\section{Energy Storage Capacitor}

To cumulate the DC voltage, which is delivered by the rectifier, a storage capacitor is used. The value of the capacitor determines the amount of energy which can be stored. The leakage current of the capacitor should be as small as possible. Smaller capacitors charge more quickly, but lead to shorter operation cycles. Larger capacitors charge more slowly, but provide higher operation cycles. Because of this, the value of the storage capacitor depends on the application. The following equation can be utilized to estimate the necessary capacitor value.

$$
C=15 \frac{1}{V^{2}} V_{\text {out }} I_{\text {out }} t_{\text {on }}
$$

$V_{\text {out }}$ and $I_{\text {out }}$ are the voltage and average current at the output of the DC-to-DC converter and $t_{\text {on }}$ is the on-time of $V_{\text {out }}$. For the proposed harvester, $\mathrm{C}$ has the value of $400 \mu \mathrm{F}$, which leads to an on-time of approximately $4 \mathrm{~ms}$ at an output voltage of $1.8 \mathrm{~V}$ and an average output current of $3.7 \mathrm{~mA}$. These parameters are suitable to power a wireless low power sensor system consisting of a MCU and a sensor.

\section{Comparator}

A comparator is an electrical circuit that compares two different analog voltages and outputs a digital voltage that indicates which one of the compared voltages has the larger value. The comparator is needed to make sure that the energy which is stored at the storage capacitor cumulates until a certain voltage $\left(V_{c a p, \max }\right)$ is reached. After exceeding this voltage, the comparator output switches to high which results in discharging the storage capacitor until a defined voltage $\left(V_{c a p, \min }\right)$ is reached. This behavior is visualized by fig. 6 .

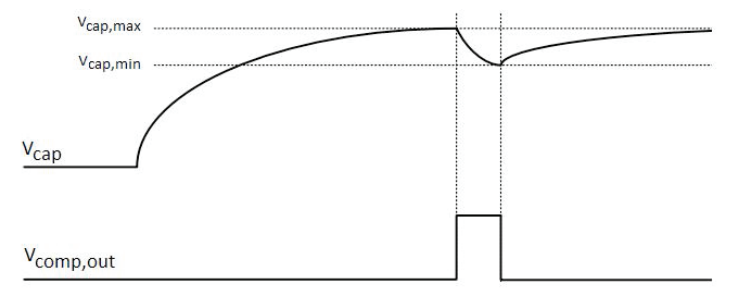

Fig. 6. Output voltage of the comparator depending on the storage capacitor voltage.

For the proposed RF energy harvesting circuit, we use the Maxim MAX9064 low power comparator. He has a minimum supply voltage of $1 \mathrm{~V}$ and consumes maximal only $700 \mathrm{nA}$ supply current. This low-voltage capability makes the comparator very attractive to passive wireless devices. The comparator has an internal $0.2 \mathrm{~V}$ reference voltage. In the proposed RF energy harvesting system, the supply voltage is provided by the voltage which is applied at the storage capacitor. The two switching voltages at which the comparator switches on and off can be dimensioned via external resistors. The resistor dimensioning is explained in detail at [3]. The external resistor dimensioning ensures that the two switching 
voltages occur at $V_{\text {cap, } \max }=1.27 \mathrm{~V}$ and $V_{\text {cap, min }}=0.940 \mathrm{~V}$. The difference of this voltages is $330 \mathrm{mV}$ and is called hysteresis bandwidth. In addition, the comparator has the purpose to prevent the circuit from overvoltage, because the maximum voltage $V_{c a p \text {, } \max }$ at the storage capacitor, which supplies the comparator and the DC-to-DC converter, is never exceeded, independent from the input power. If the input power increases, the operation cycles are just carried out more quickly due to the faster charging of the capacitor, but the maximum voltage at the storage capacitor does not change. [4]

\section{DC-to-DC Converter}

A DC-to-DC converter is an electronic circuit that is able to convert $\mathrm{DC}$ voltage from one level to another. For our harvester, we use the LTC3526L DC-to-DC converter from Linear Technology. He has the task to increase the voltage which is applied at the storage capacitor $(940 \mathrm{mV}-1.27 \mathrm{~V})$ to a $\mathrm{DC}$ voltage of $1.8 \mathrm{~V}$ during one operation cycle. The output voltage can be adjusted between $1.5 \mathrm{~V}$ and $5.25 \mathrm{~V}$ by an external resistor divider tap. The device has an input voltage range between $0.5 \mathrm{~V}$ to $5 \mathrm{~V}$ and is supplied by the storage capacitor. [5]

\section{Complete RF Energy Harvesting System}

The RF energy harvesting circuit proposed in this work has been designed with CadSoft EAGLE and fabricated using PCB technology. The maximum harvesting range of the system is approximately $2 \mathrm{~m}$ and the minimum necessary input power is $-6 \mathrm{dBm}$. Fig. 7 shows the voltage at the output of the harvester (lower graph) and the voltage at the storage capacitor (upper graph) during one operation cycle. These voltages have been measured with an oscilloscope. It can be seen at the figure that the voltage at the storage capacitor decreases from $1.27 \mathrm{~V}$ to $940 \mathrm{mV}$ which are the two threshold voltages determined by the comparator resistor dimensioning. During the discharge of the storage capacitor the DC-toDC converter outputs a DC voltage of $1.8 \mathrm{~V}$ for a time of $4 \mathrm{~ms}$. This is indicated by the two vertical lines at fig. 7 . Within the on-time of the DC-to-DC output voltage, the load, which is typically a wireless sensor system, is powered and able to execute one operation cycle. Such cycle consists for example of the determination and transmission of temperature and humidity values. After operation, the sensor system waits on standby until the next operation cycle begins.

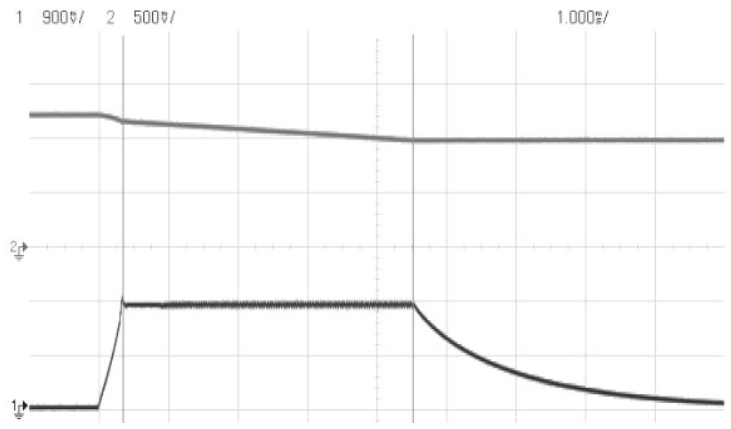

Fig. 7. Vcap and Vout over time during one cyle

\section{Conclusion and Future Work}

In this paper, a RF energy harvesting system for the $868 \mathrm{MHz}$ band is presented. It can be used to energize low power devices, e.g. wireless sensor systems. The complete RF energy harvesting system can operate at input powers of at least $-6 \mathrm{dBm}$ and is optimized for a frequency of $866.6 \mathrm{MHz}$ and has a maximum harvesting range of $2 \mathrm{~m}$. It outputs an energy of up to $60 \mu \mathrm{J}$ during one operation cycle.

One future task of this work is the investigation of the behavior of the presented RF harvester circuit if it is integrated into several building and insulation materials, e.g. reinforced concrete or steel wool. Integrated into these materials, the RF energy harvesting circuit should be able to power a wireless sensor system, which measures and transmits the temperature and humidity values of these materials.

\section{References}

[1] K. Dembowski, Energy Harvesting fuer die Mikroelektronik, pp. $24 \mathrm{ff}, 1$ st edition, VDE Verlag, Berlin, 2011.

[2] C. Bowick, J. Blyler, C. Ajluni, RF Circuit Design, page 71, Newnes, $2^{\text {nd }}$ edition, 2007

[3] Maxim Integrated. (2005, Sep.) Application Note 3616: Adding Extra Hysteresis to Comparators. [Online]. Available: http://www.maximintegrated.com/appnotes/index.mvp/id/3616

[4] Maxim Integrated. (2014, Mar.) Ultra-Small, LowPower Single Comparators in 4-Bump UCSP and 5-SOT23. [Online]. Available: www.maximintegrated.com/datasheet/index.mvp/i $d / 5823$

[5] Linear Technology Corporation. (2014, Mar.) LTC3526L/LTC3526LB. [Online]. Available: www.linear.com/product/LTC3526L 